\title{
BMJ Open E-health ecosystem with integrated and stepped psychosocial services for breast cancer survivors: study protocol of a multicentre randomised controlled trial
}

\author{
Cristian Ochoa-Arnedo (D) ,1,2,3 Joan Carles Medina (D) , ${ }^{1,2,4}$ Aida Flix-Valle (D) , 1,2,3 \\ Dimitra Anastasiadou (1) ${ }^{1,3}$
}

To cite: Ochoa-Arnedo C, Medina JC, Flix-Valle A, et al. E-health ecosystem with integrated and stepped psychosocial services for breast cancer survivors: study protocol of a multicentre randomised controlled trial. BMJ Open 2021;11:e041548. doi:10.1136/ bmjopen-2020-041548

- Prepublication history and additional material for this paper is available online. To view these files, please visit the journal online (http://dx.doi.org/10. 1136/bmjopen-2020-041548).

Received 12 June 2020 Revised 02 February 2021 Accepted 10 February 2021

Check for updates

(C) Author(s) (or their employer(s)) 2021. Re-use permitted under CC BY-NC. No commercial re-use. See rights and permissions. Published by BMJ.

${ }^{1}$ Programa E-Health ICOnnecta't and Unidad de Psico-Oncología, Institut Català d'Oncologia, Barcelona, Spain

${ }^{2}$ Universitat de Barcelona, Barcelona, Spain

${ }^{3}$ Psico-oncologia, Recerca en serveis sanitaris en càncer, Institut d'Investigació Biomèdica de Bellvitge (IDIBELL), Hospitalet del Llobregat, Barcelona, Spain ${ }^{4}$ Department of Psychology and Educational Sciences, Universitat Oberta de Catalunya, Barcelona, Spain

Correspondence to Dr Cristian Ochoa-Arnedo; cochoa@iconcologia.net

\section{ABSTRACT}

Introduction Psychosocial interventions for patients with breast cancer $(\mathrm{BC})$ have demonstrated their effectiveness at reducing emotional distress and improving quality of life. The current digitisation of screening, monitoring and psychosocial treatment presents the opportunity for a revolution that could improve the quality of care and reduce its economic burden. The objectives of this study are, first, to assess the effectiveness of an e-health platform with integrated and stepped psychosocial services compared with usual psychosocial care, and second, to examine its cost-utility

Methods and analysis This study is a multicentre randomised controlled trial with two parallel groups: E-health intervention with integrated and stepped psychosocial services vs usual psychosocial care. An estimated sample of 338 patients with $B C$ in the acute survival phase will be recruited from three university hospitals in Catalonia (Spain) and will be randomly assigned to one of two groups. All participants will be evaluated at the beginning of the study (T1: recruitment), 3 months from T1 (T2), 6 months from T1 (T3) and 12 months from T1 (T4). Primary outcome measures will include number of clinical cases detected, waiting time from detection to psychosocial intervention and proportion of cases successfully treated in the different steps of the intervention, as well as outcomes related to emotional distress, quality of life, post-traumatic stress and growth, treatment adherence and therapeutic alliance. Secondary outcomes will include the acceptability of the platform, patients' satisfaction and usability. For the cost-utility analysis, we will assess quality-adjusted life years and costs related to healthcare utilisation, medication use and adherence, work absenteeism and infrastructure-related and transport-related costs.

Ethics and dissemination This study was approved by the Ethics committee of the Institut Català d'Oncologia network in Hospitalet, Spain. Findings will be disseminated through peer-reviewed journals, reports to the funding body, conferences among the scientific community, workshops with patients and media press releases.

Trial registration number Online Psychosocial Cancer Screening, Monitoring and Stepped Treatment in Cancer Survivors (ICOnnectat-B),NCT04372459.
Strengths and limitations of this study

- The sample size was calculated taking into account the high dropout rate commonly reported in e-health interventions; to deal adequately with this limitation, an extra $25 \%$ of patients will be recruited

- The study focuses on the specific group of patients with $\mathrm{BC}$ and consequently difficulties may be encountered in generalising results to other cancer populations.

- This trial is designed as a multicentre study and uses a rigorous methodology.

- Collecting data on healthcare utilisation and its costs and work absenteeism at different time points will allow a comprehensive economic evaluation of the e-health intervention.

- Intention-to-treat analyses will be performed using linear mixed models to examine the longitudinal changes of participants according to their assigned treatment group.

\section{BACKGROUND}

It is estimated that there are currently over 100 million cancer survivors worldwide, a number that is constantly increasing. ${ }^{1}$ More specifically, according to the Spanish Society of Medical Oncology, ${ }^{2}$ one in eight women in Spain will present breast cancer (BC) at some point in their lives, a disease that has a 5-year relative survival rate of $89.2 \%$. However, an increase in survival rates does not necessarily imply greater well-being, since several studies have shown that cancer diagnosis and its treatment cause physical limitations, psychosocial problems, work-related challenges and poor quality of life among survivors. ${ }^{34}$ In particular, psychological distress is found among almost $40 \%$ of individuals who have gone through an oncological process ${ }^{5}$ and evidence shows that when it is treated adequately, it is associated with better health outcomes and higher survival rates. ${ }^{67}$ However, only a small proportion of survivors is currently screened 
and referred to the proper health professionals to receive psychosocial care, and the waiting time to receive such treatment is often long; for example, workload and time constraints have been mentioned as important barriers to treatment delivery by health professionals. ${ }^{8}$

In recent years, e-health has emerged as an innovative resource to expand the availability and reach of psychological treatments for cancer survivors. At the same time, e-health has been able to mitigate concerns about anonymity and barriers associated with long waiting lists at hospitals or work, and time and mobility restrictions. The benefits of e-health in promoting change in healthrelated behaviours and knowledge, as well as in quality of life and other clinical outcomes, have been well documented in the literature. ${ }^{9-11}$ Specifically, there is empirical evidence examining the effectiveness of the independent use of web-based platforms for stress management, coping and behavioural monitoring in $\mathrm{BC}^{12-14}$ online educational platforms ${ }^{15-17}$ and online support groups. ${ }^{18-21}$ However, evidence-based e-health interventions specifically designed for patients with BC that offer personalised and stepped interventions adapted to patients' needs are lacking, as are studies assessing their clinical efficacy, costeffectiveness and feasibility. ${ }^{10}$

In this context, the e-health unit and the psychooncology department of the Institut Català d'Oncologia (ICO) developed the programme ICOnnecta't. ${ }^{22}$ This programme is the first innovative e-health ecosystem aimed at building online communication spaces and fostering healthy experiences among patients ith BC. The programme also integrates a psychosocial programme of four steps that are ranked according to patient complexity. This programme was initially implemented in 2019 in three centres of the ICO (Hospitalet, Badalona and Girona) (http://ico.gencat.cat/ca/__institut/ centres/) and 6 months later it was expanded in Europe (Instituto Português de Oncologia de Coimbra, Portugal, and Szpital Mikolaja Kopernika w Łodzi, Poland) with the name 'ONCOMMUN' (https:/ / oncommun.eu), a project supported by the prestigious European Institute of Innovation and Technology. Since 2020, the project has been implemented at two more Spanish institutions (Hospital General Universitario Gregorio Marañón in Madrid, and Hospital de la Santa Creu i Sant Pau in Barcelona).

ICOnnecta't guarantees the provision of the correct screening, monitoring and support in health education, and adherence to treatments, and provides emotional and psychosocial support during the different transitions that take place in the oncological process, through evidence-based, stepped and integrated services. The first level of care of ICOnnecta't is an online screening and monitoring tool (level 1), followed by a cancer survivors' campus with online educational resources to increase knowledge about the disease (level 2), a psychosocial support community where patients and professionals can interact, in the form of a private social network (level 3) and a psycho-oncological group treatment through videoconference (level 4).
The purpose of this study is to conduct a multicentre randomised controlled trial (RCT) with an estimated sample of 338 patients with BC. In this RCT, participants in the experimental group will use the e-health ecosystem ICOnnecta't, while those in the control group will follow the usual psychosocial treatment (ie, one individual 1-hour session with a clinical psychologist every month). Then, effectiveness and cost-utility analyses will be performed. To this end, we have the following specific objectives:

- To examine the effectiveness of the e-health platform compared with usual psychosocial care in terms of: (1) waiting time for detection, delivery of psychosocial care and resolution of clinical cases, (2) change in psychological variables including emotional distress, post-traumatic stress and growth, therapeutic alliance and quality of life and (3) users' satisfaction with the platform and its usability.

- To compare the cost-utility balance between the e-health platform and the usual psychosocial care in terms of QALYs, time lapse for return-to-work, pharmacological adherence and healthcare utilisation with all the related costs.

The implementation of the online intervention programme is expected to result in a significant improvement in the primary and secondary outcome measures compared with the control group. More specifically:

- The e-health platform should lead to significantly greater improvements (differences between $\mathrm{T} 1$ and $\mathrm{T} 2$, which should also be maintained at T3 and T4) in the primary outcome variables, including waiting time for detection, intervention and resolution of mild and moderate clinical cases as well as emotional distress, post-traumatic stress and growth, therapeutic alliance and quality of life, in comparison to usual care.

- The e-health intervention should prove to be more cost-effective compared with usual care in terms of QALYs, pharmacological adherence, work absenteeism and costs related to healthcare utilisation.

- The e-health platform should show satisfactory levels of acceptability and usability by users.

\section{METHODS AND ANALYSIS \\ Design}

The study proposes a multicentre superiority RCT with two parallel groups (online integrated and stepped psychosocial care vs usual psychosocial care) with 1:1 allocation. The study design will contain 2 (treatment conditions) $\times 4$ (follow-up assessments) factors. The Consolidated Standards of Reporting Trials 2010 statement: extension to pilot and feasibility trials has been followed in the planning of the study and reporting of the protocol. ${ }^{23}$

\section{Patient and public involvement}

The study follows a responsible research and innovation approach, according to which the research question was shared, coconstructed and informed to our cancer patient committee and collaborating non-profit 
cancer associations. Patients were actively involved in the design of the ecosystem through cocreation/codevelopment activities. Patients will be indirectly involved in the recruitment, informing and encouraging other patients to participate. Finally, results will be disseminated to study participants and general public through citizen engagement meetings.

\section{Sample}

The total sample will be approximately 338 patients with a diagnosis of BC during the acute survival phase (from 6 weeks' postdiagnosis until the end of primary oncological treatment) from three centres of the ICO network in Catalonia (Hospital Duran i Reynals in Hospitalet, Hospital Germans Trias i Pujol in Badalona and Hospital Josep Trueta in Girona).

\section{Inclusion criteria}

1. Patients aged $\geq 18$ years with BC, within 6 weeks after diagnosis.

2. Having online access and a user-level knowledge of the internet.

3. Understanding of Spanish language.

\section{Exclusion criteria}

1. Major depressive episode.

2. Significant autolytic ideation.

3. Showing symptoms of psychosis or substance abuse.

4. Intellectual disability or any major illness seriously affecting their cognitive performance (eg, neurological disorders).

Considerations for access to the internet for the experimental group only: It will be mandatory to have access to the internet using a proper terminal (eg, mobile, tablet or personal computer) that meets the minimum requirements to participate in the study. This includes access to the terminal in a private place and having a microphone and a webcam. We will lend a webcam, a microphone, or a tablet to those patients who do not have these resources. This first-stage contact with patients constitutes a brief digital reception focused only on the installation of the App and a little road trip ( $5 \mathrm{~min}$ ) in its interface and dynamics, avoiding any potential digital, educational or psychosocial empowering which could be a cofounder.

\section{Procedure and randomisation}

All material with information related to the study (research protocol, informed consent, patient information sheet) has been approved by the Ethics committee headquarters of the ICO network in Hospitalet (PR289/19).

Participants will be recruited in the oncology and radiotherapy services of the three participating hospitals, with variation in the number of recruited patients depending on each hospital's care volume.

Selection and invitation to participate in the study will take place when the patients first visit the nursing staff assigned to them, immediately after visiting their oncologist, at which point the diagnosis and care plan will have been shared with them. The nurse will introduce the study and explain its objectives. Next, for women interested in taking part in the study, face-to-face meetings with psychologists in our research team will take place, in which the inclusion criteria will be verified through a brief interview, the study objectives will be presented and the written informed consent will be signed in the case of acceptance. Then, a randomisation procedure will take place to assign the treatment groups (experimental or control group). This randomisation will be carried out through a list of randomly generated numbers on a computer system using IBM SPSS V.24 software ${ }^{24}$ by an independent researcher blind to the research questions and treatment conditions. The psychologist carrying out the recruitment interview will be responsible for communicating the assignment of the study group. Given the nature of both treatment modalities, neither patients nor health professionals will be blind to intervention assignment.

Patients from the experimental group will be given oral and written instructions about the basic functioning of the e-health platform and the applications will be downloaded onto their mobile phones. In turn, patients from the control group will be told that a mental health professional at their institution will contact them to arrange the first face-to-face visit. Then, each group of patients will receive their corresponding treatment for a period of 12 months.

All participants will be evaluated through online surveys at the beginning of the study (T1: recruitment), 3 months from T1 (T2), 6 months from T1 (T3) and 12 months from T1 (T4), regardless their assigned arm. However, only participants from the experimental group will be asked to complete the System Usability Scale (SUS) and the digital emotional state thermometer, since these are associated with the functionality of the e-health stepped programme. To optimise participant retention and complete follow-up, reminder mails will be sent to all participants at T2, T3 and T4. Finally, if a significant worsening on patient's psychological well-being is identified during the whole procedure, a face-to-face visit with an expert in psycho-oncology of one of the ICO Hospitals will be scheduled for her, to assess whether or not she will have to discontinue her participation in the trial. Figure 1 presents the procedure and the timeline of the study and table 1 provides a detailed description of the methodology with a definition of the study variables and their assessment tools.

\section{Study conditions and data collection \\ E-health ecosystem with integrated and stepped psychosocial services}

Survivors allocated to this intervention will be monitored by the system for early detection of possible psychosocial needs, allowing the delivery of integrated and stepped care, when necessary. The programme ICOnnecta $\mathrm{t}^{22}$ features four levels, increasing in intensity of psychosocial care as patients move from one level to the next. Members of the research team will coordinate the monitoring of patients through the e-health platform. Psychologists performing the online psychosocial interventions in the e-health platform will receive specific training. 
Invitation to participate during visit with nursing staff

Study presentation, Informed Consent, Brief interview for eligibility

Exclusion

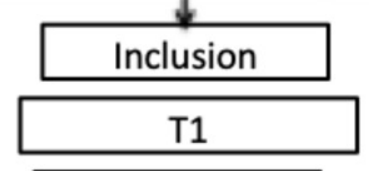

Randomization

4

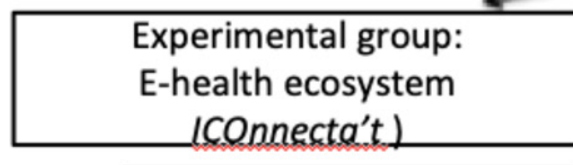

Training with e-health ecosystem
Control group:

Usual psychosocial treatment

\section{Contact to arrange first} face-to-face visit



Figure 1 Timeline and procedure.

The four steps break down as follows:

\section{Step 1: screening and monitoring}

The screening and monitoring of several psychosocial variables will be carried out through the central mobile application of the programme (App ICOnnecta't). Healthcare professionals will schedule alerts on their patients' devices requesting them to complete specific questionnaires, and in this way, the evolution of their medication adherence (concomitant pharmacological treatment), emotional distress and well-being, among other health indicators (see table 1 ), as well as any spontaneously reported adverse event will be monitored on a regular basis. Likewise, patients will have at their disposal an encrypted messaging system to communicate adverse effects, abandonment or worsening of their mood to their healthcare professional. The indicator for moving to the next and more intensive level of psychosocial care will be scores $\geq 6$ (positive screening) on the emotional distress thermometer for two consecutive weeks. Women with positive screening results will be asked to answer a standardised instrument for the detection of clinical distress, the Hospital Anxiety and Depression Scale (HADS). Patients whose scores on this scale confirm clinical distress (HADS scores $>10$; positive monitoring) will be scaled up to the second level of care. The same procedure will be carried out for movements from the second to third level and from the third to fourth level, although participants will stay in each step for at least 2 weeks. It is worth declaring that the abovementioned procedure is an adaptation of the stepped care programme for cancer patients described by Jansen et $a l^{25}$ In addition, all movements between levels will be preceded by a videoconference with a psychologist in the research team to accompany and guide patients throughout the process.

\section{Step 2: campus: psychoeducation and health education}

The ICOnnecta't Campus forms the second level of psychosocial care. This is an educational platform on Moodle via which patients can consult videos and online resources, co-constructed between health professionals and patients, containing reliable and rigorous information related to the oncological process. This information will be divided into thematic blocks, each one describing challenges found at the different stages of the oncological process, including emotions, medical treatments, personal relationships and healthy habits. After accessing each resource, users will be asked to complete a questionnaire to appraise the health knowledge acquired. The same questionnaire will be administered to participants in the control group. 
Table 1 Instruments for measuring clinical efficacy, cost-effectiveness and adherence to the online stepped intervention

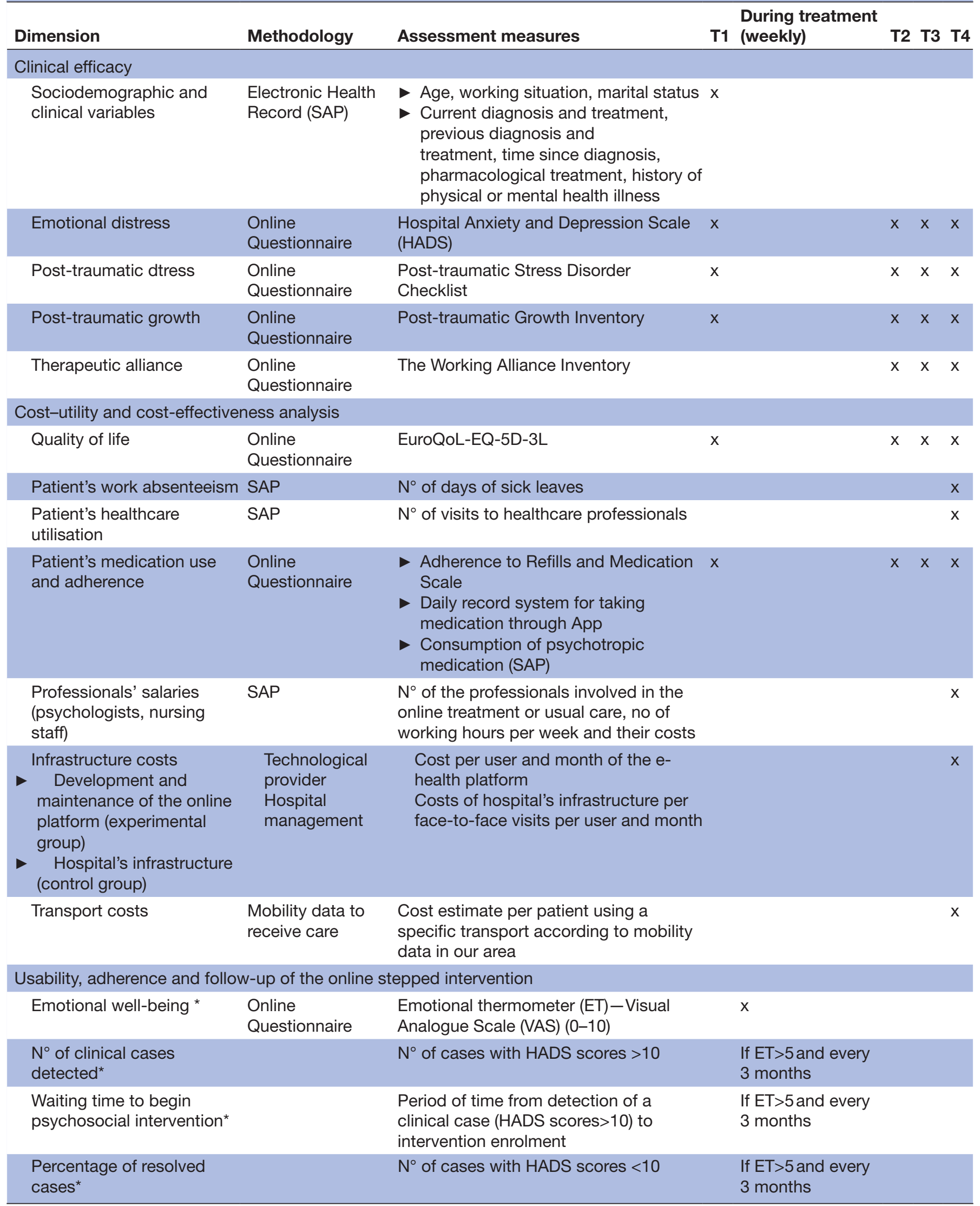

Continued 


\begin{tabular}{|c|c|c|c|c|c|c|}
\hline Dimension & Methodology & Assessment measures & T1 & $\begin{array}{l}\text { During treatment } \\
\text { (weekly) }\end{array}$ & T2 & T3 T4 \\
\hline Usability and satisfaction & $\begin{array}{l}\text { Online } \\
\text { Questionnaires }\end{array}$ & $\begin{array}{l}\text { - System Usability Scale } \\
\text { - VAS }(0-10)\end{array}$ & & $\begin{array}{l}\text { Third week after } \\
\text { registration }\end{array}$ & & $x$ \\
\hline
\end{tabular}

*These variables will be measured in patients from the experimental group only.

EuroQoL-EQ-5D-3L, European Quality of Life-5 Dimensions-3 Levels; SAP, Servicio de Atención al Paciente.

\section{Step 3: psychosocial support community}

If the patient does not improve within 2 weeks during step 2, she will be contacted by a psychologist from the research team through a videoconference and be offered the option to participate in the online community of psychosocial support for patients ith BC (App Comunitats). This online community aims to create a digital therapeutic alliance between patients, and between patients and health professionals. This community is anonymous and is supervised by a psycho-oncologist, an oncological nurse and a BC survivor mentor with experience in the use and support of virtual communities. Specifically, through this private social network, participants can choose from 12 cancer-related discussion forums and share with the community any health-related question that worries them, thus fostering debate and stimulating peer-support.

\section{Step 4: intensive online group psychotherapy}

If the patient does not improve within 2 weeks during step 3 , she will be offered the option to participate in weekly group-based psychotherapy (App ICOnnecta't Videoconsulta), which is based on the Group Positive Psychotherapy programme for cancer survivors ${ }^{26} 27$ and adapted for patients under primary oncological treatments. Group positive psychotherapy has been compared with a waiting-list control group in a previous study ${ }^{28}$ and to other evidence-based interventions ${ }^{29}$ and showed better results in terms of patients' psychological well-being. This step will compromise 8 weekly 90 min sessions led by a clinical psychologist (an expert in psycho-oncology) with a follow-up 3 months later. In addition, patients will be offered an online appointment with a psychiatrist to assess the need for psychopharmacological treatment if emotional distress persists.

\section{Psychosocial treatment as usual}

This active control group will receive the standard psychosocial treatment for cancer survivors in the participating centres, led by a clinical psychologist. In a recent agreement (2018) regarding the standard psychosocial treatment offered by the three participating hospitals, it was established that the average psycho-oncological treatment offered consisted of 6-9 individual, 60 min sessions focused on emotional support and psychoeducation during the first year of primary cancer treatment. In order to be more systematic in the programme design and ensure homogeneous 'treatment as usual' in the three participating hospitals, all hospitals committed to carrying out 8 monthly psycho-oncological visits of $45-60$ min per participant.

\section{Measurements}

\section{Clinical efficacy measures}

Sociodemographic and clinical data will be obtained from the hospital's electronic health record (Servicio de Atención al Paciente, SAP).

\section{Emotional distress}

The $\operatorname{HADS}^{30}$ is an instrument designed to detect the presence and severity of anxiety and depression in people with physical illnesses. It has been validated in Spanish among a sample of patients with cancer, ${ }^{31}$ and showed good reliability $(\alpha=0.82$ and $\alpha=0.84$ for anxiety and depression subscales, respectively). It consists of 14 items, 7 for anxiety and 7 for depression, rated on a four-point Likert scale (range $0-3$ ), with the total score ranging from 0 to 42. Higher scores indicate a greater level of distress.

\section{Post-traumatic stress}

The Post-traumatic Stress Disorder Checklist for Diagnostic and Statistical Manual of Mental Health Disorders $(\mathrm{DSM}-\mathrm{V})(\mathrm{PCL}-5)^{32}$ is a 20 -item self-report questionnaire designed to assess post-traumatic stress disorder, according to the DSM-V. ${ }^{33}$ The questionnaire demonstrated high internal consistency $(\alpha=0.94)$ and good testretest reliability $(\mathrm{r}=0.82)$. The official Spanish translation of the instrument was provided by the National Center for Post-Traumatic Stress Disorder (https://www.ptsd.va. gov/).

\section{Post-traumatic growth}

The PTGI $^{34}$ is a 21-item instrument that assesses positive changes experienced after a trauma. Its Spanish validation among cancer patients by Costa-Requena and Gil ${ }^{35}$ showed excellent internal consistency $(\alpha=0.95)$. We also incorporate in our analysis of PTG, the new knowledge and methods to interpret the productive and illusory part of this potential therapeutic response. ${ }^{36-38}$ 


\section{Therapeutic alliance}

The Working Alliance Inventory (WAI) ${ }^{39-41}$ is a questionnaire designed to assess the three components of the therapeutic alliance defined by Bordin ${ }^{42}$ : therapistpatient agreement regarding the goals of psychotherapy; therapist-patient agreement regarding the tasks to be completed in psychotherapy and therapist-patient bond. The patient form (WAI-P) and therapist form (WAI-T) of the WAI consist of 36 items organised into three subscales (Goal, Task, Bond) of 12 items each. The instrument has been validated in the Spanish population, ${ }^{43}$ showing excellent reliability of both forms (WAI-P and WAI-T) $(\alpha=0.96)$.

\section{Cost-utility and cost-effectiveness analysis \\ Quality of life}

The EuroQoL-EQ-5D-3L $\mathrm{L}^{44}$ is a standardised instrument used as a health outcome measure for economic evaluation studies. It consists of five dimensions: mobility, self-care, usual activities, pain/discomfort and anxiety/ depression. Each dimension has five levels: no problems, slight problems, moderate problems, severe problems and extreme problems. The Spanish validation of the instrument was carried out by Badia $e t \mathrm{al}^{45}$

\section{Healthcare utilisation}

The total number of visits to each healthcare professional (ie, oncologist, nursing staff, psychologist, psychiatrist, social worker, emergency department) will be assessed through the hospital's SAP.

\section{Medication use and adherence}

The Adherence to Refills and Medications Scale (ARMS) ${ }^{46}$ was developed to measure medication adherence among polypathological patients. It consists of 12 questions: 8 aimed at assessing the patient's ability to properly administer the medication and 4 regarding its correct intake based on a four-point Likert scale (range from $1=$ never to $4=$ always), with lower scores indicating better adherence. The instrument has been validated in Spanish (ARMSe) ${ }^{47}$ showing satisfactory psychometric properties. Apart from the online questionnaire, a daily medication record will be obtained through the monitoring system of the ICOnnecta't application.

\section{Work absenteeism}

The number of days of sick leave per patient will be assessed through the hospital's SAP.

\section{Professionals' salaries}

The number of professionals involved in the online treatment or usual care as well as their number of working hours per week and their costs will be assessed through the SAP.

\section{Infrastructure costs}

The costs per user and per month for the development and maintenance of the online platform (experimental group) and the costs per user and per month for the hospital's infrastructure to guarantee face-to-face visits with patients (control group) will be obtained from each provider.

\section{Transport costs}

An estimate of the cost per patient of using a specific type of transport will be obtained through official governmental mobility data from the Barcelona and Girona metropolitan areas.

\section{Usability, adherence to and efficacy of the online stepped intervention \\ Emotional well-being}

Emotional well-being will be measured weekly through an emotional state thermometer based on a Visual Analogue Scale (VAS) in the ICOnnecta't application, only for the patients in the experimental group.

Number of clinical cases

The number of clinical cases (those obtaining HADS scores $>10$ ) will be recorded during treatment.

\section{Waiting-time to begin psychosocial intervention}

The period of time from the detection of a clinical case (HADS scores $>10$ ) to enrolment in the intervention will be assessed.

\section{Percentage of resolved cases}

The eventual resolution of a clinical case (if patient reports HADS $<10$ ) will also be measured.

\section{Health education}

Scores on health education questionnaires developed by our research group will be obtained from the Campus of ICOnnecta't throughout the duration of treatment through single measurements per topic. For the control group, health knowledge will be assessed using the same health education questionnaires in their online format.

\section{Usability}

The $\mathrm{SUS}^{48}$ is a 10 -item questionnaire that will be used to measure the usability of the e-health platform as perceived by users in the experimental group.

\section{Satisfaction}

The satisfaction of users in the experimental group with the platform will be assessed through a VAS (0-10).

\section{Sample size calculation}

Sample size was calculated with the support of the GLIMMPSE software after having consulted results from the previous RCT conducted by van de Berg $e t a l^{14}$, which evaluated the effectiveness of the BREATH self-management platform for patients with BC. To demonstrate a smallto-medium effect size of $\mathrm{d}=0.40$ between both arms in emotional distress (HADS), 135 patients will be needed in each treatment group (power of $80 \%$, significance level of $5 \%)$. This estimation has considered the site $\times$ treatment interaction and assumes a variability scale factor of 0.5 , given the similarities between centres (they all 
pertain to the same institution). Allowing for a dropout rate of $25 \%$, a total of 338 patients need to be recruited. With an annual intake of $1800 \mathrm{BC}$ survivors in the three centres during diagnosis and primary oncological treatment, $30 \%$ of whom present significant psychological distress and $50 \%$ of whom would be willing to cooperate, the feasibility of the study is guaranteed.

\section{Data management}

All data in paper form related to the trial, including the health professionals' paper notes, self-report questionnaires and informed consent forms, will be securely stored in a key-protected cupboard in the principal investigator's office at ICO. Regarding online data, these will be saved on an encrypted external hard drive stored in the same cupboard. All data that support the findings of the study will be stored for a minimum of 5 years and be available on request from the corresponding author. Only authorised researchers directly involved in the study will have access to this information.

After obtaining the signed informed consent from participants, a unique alphanumeric code will be allocated to each one of them so that analyses can be conducted anonymously. The database that links participants to their codes will be securely stored on a passwordprotected server that will only be accessible to the research team. Finally, the information collected through the e-health platform will be stored in a secure collaborative cloud compliant with the latest version of the European General Data Protection Regulation (EC/2016/679). An anonymised dataset will be uploaded to the Open Science Framework platform (https://osf.io/), once deleted all personal and identifiable information.

\section{Statistical analyses}

Descriptive methods will be used to analyse sociodemographic and clinical variables. Between-group analyses will be conducted for baseline differences using Pearson's $\chi^{2}$ test or Fisher's exact test for categorical data and Student's t-test or the Mann-Whitney U test for continuous variables, depending on the normality of the distribution. The normality of the distribution of the variables will be assessed using the Kolmogorov-Smirnov test.

Intention-to-treat analyses will be performed using generalised linear mixed models to test the effect of interventions (experimental vs control group) on distress (HADS scores), post-traumatic stress (PCL-5) and PTGI over time (T1-T4), while effect sizes (Hedges' g) will be reported. This procedure considers the multiple dependence between repeated measures while preserving analytic power. Later, the effect of the centre $\times$ intervention group interaction will also be tested. Finally, the influence of age in the results will be assessed, since its relationship with digital literacy presents it as a potential confounder. Models will be built parsimoniously, with maximum likelihood as the estimation method, and Little's Missing Completely At Random (MCAR) test will be used to ascertain whether data is missed completely at random before deciding if imputation is needed.

For the cost-effectiveness analyses, results from the EQ-5D-3L will be used to generate the index of life years adjusted for quality of life. The cost of each intervention (e-health intervention vs psychosocial treatment as usual) will be assessed in terms of the psycho-oncologist's involvement and his/her salary, the number of online or face-to-face sessions, infrastructure costs, as well as patient's medication use and adherence, work absenteeism and transport costs. Then, a specific analysis of cost-effectiveness between the e-health intervention and the usual psychosocial treatment will be calculated according to the target outcomes of the study: reduction of emotional distress, improvement of quality of life, improvement of post-traumatic growth and reduction of post-traumatic stress.

Analyses will be conducted using SPSS V.24.0 $0^{24}$ and HLM V.7.0.3 ${ }^{49}$ supported by the expertise of Universitat Oberta de Catalunya eHealth Centre in secure health information management.

\section{DISCUSSION}

This multicentre RCT will assess the clinical efficacy and cost-utility of a digital health ecosystem that integrates a four-stepped psychosocial programme that is adapted to the specific needs of $\mathrm{BC}$ survivors and organised according to their level of complexity. This is an important strength of this study because, to our knowledge, there is no previous evidence supporting the efficacy of online integrated and stepped interventions in this population.

Regarding the limitations of the study, we must acknowledge that it focuses on the specific group of patients with BC. As a result, we may encounter difficulties in generalising results to other cancer populations. However, efforts by our research team are being made to scale the platform to patients with oncohaematological diseases, advanced lung cancer and other malignancies in different geographical areas.

The design of the study brings along some methodological difficulties, especially the risk of a high dropout rate over time. To address this challenge, we will include an extra $25 \%$ of patients in the trial to ensure that the statistical power will be maintained despite patient attrition.

Although the acceptability of online treatments is growing $^{11}$ and recent studies indicate an increase in patients' use of health-related information on internet and in participation in interactive communities, ${ }^{50}$ the age of participants and their respective digital literacy may condition access to new technologies. As such, we will explore the potential confounding effect of age in data analyses. In addition, we will provide patients of all ages with a technical support service that will be able to train them on how to use the platform and solve any technological issue. In addition, the team has produced video tutorials on the correct use of each of the four steps of the platform. Access to technological devices could also be a 
limitation, but we intend to solve that by lending patients the necessary devices (ie, webcam, tablet, microphones, headphones).

If the findings of this research prove to be positive, then the project may lead to critical advances towards an integrated model of psychosocial care in cancer that could provide fully connected, personalised psychosocial support for the specific needs of each survivor. Such advances have the potential to act as a turning point in the delivery of patient-centred interventions using new technologies and address one of the main challenges posed by today's digital society in serious diseases, such as cancer: how to build a therapeutic alliance between citizens and health providers to manage chronic conditions in a sustainable way.

\section{Ethics and dissemination}

Ethical approval of the study protocol and the informed consent forms was received and approved (PR289/19) from 'ICO Hospitalet review board' (20/02/2020) with respect to the scientific content of the protocol and its compliance with applicable research and human subjects' regulations (see ONCOMMUNITIES informed consent on online supplemental material 1. Patients' participation is with fully informed written consent, which is revocable at any time.

Findings will be disseminated at a national and international level through peer-reviewed journals, reports to the funding body, conferences among the scientific community, workshops with patients and media press releases.

\section{Twitter Cristian 0choa-Arnedo @Cristian8apsy}

Acknowledgements We thank CERCA Programme Generalitat de Catalunya for institutional support. We would also like to thank all patients and health professionals for their willingness to participate in the study, for whom several dissemination activities to share trial results are expected.

Contributors All coauthors of this study met the ICMJE criteria for authorship. In specific, CO-A conceived the study. CO-A, JCM and AF-V initiated the study design; DA reviewed it and wrote the first draft of the manuscript. All authors contributed to the refinement of the study protocol and approved the final manuscript. Authorship eligibility for this publication was determined in accordance with the European Code of Conduct for Research Integrity (https://allea.org/code-of-conduct/\#toggle-id-3).

Funding This study has been funded by the European Institute of Innovation and Technology (EIT Health) (20536-ONCOMMUNITIES: Online Cancer Support Communities). EIT Health is the largest European network for health innovation. EIT Health is supported by EIT, a body of the European Union. This work has also supported by the Carlos III Health Institute under the FIS grants PI15/01278 and PI19/01880, cofinanced by the European Regional Development Fund (ERDF) 'a way to build Europe'. This funding body has the main role in the design, collection, analysis and interpretation of data. In addition, this funding entity independently and annually audits the trial by means of an investigators' report. Through this annual report, investigators will be able to communicate important protocol modifications and the funding body will assess whether the reported modifications are minor or critical and need to be authorised. Consolidated research group: Research in health services in cancer, 2017SGR00735. The role of this funding body has been to provide methodological advice, corrections and English translations. Finally, $\mathrm{CO}-\mathrm{A}$ is the principal investigator of the study and its sponsor.

Disclaimer The role of this funding body has been the technological development of the e-health ecosystem and the first proof of concept. This funding body has a relevant role in writing the manuscript.

Competing interests None declared.

Patient consent for publication Not required.
Provenance and peer review Not commissioned; externally peer reviewed.

Supplemental material This content has been supplied by the author(s). It has not been vetted by BMJ Publishing Group Limited (BMJ) and may not have been peer-reviewed. Any opinions or recommendations discussed are solely those of the author(s) and are not endorsed by BMJ. BMJ disclaims all liability and responsibility arising from any reliance placed on the content. Where the content includes any translated material, BMJ does not warrant the accuracy and reliability of the translations (including but not limited to local regulations, clinical guidelines, terminology, drug names and drug dosages), and is not responsible for any error and/or omissions arising from translation and adaptation or otherwise.

Open access This is an open access article distributed in accordance with the Creative Commons Attribution Non Commercial (CC BY-NC 4.0) license, which permits others to distribute, remix, adapt, build upon this work non-commercially, and license their derivative works on different terms, provided the original work is properly cited, appropriate credit is given, any changes made indicated, and the use is non-commercial. See: http://creativecommons.org/licenses/by-nc/4.0/.

\section{ORCID iDs}

Cristian Ochoa-Arnedo http://orcid.org/0000-0002-4508-0951

Joan Carles Medina http://orcid.org/0000-0002-4550-2157

Aida Flix-Valle http://orcid.org/0000-0002-1962-330X

Dimitra Anastasiadou http://orcid.org/0000-0001-7544-523X

\section{REFERENCES}

1 Ferlay J, Colombet M, Soerjomataram I, et al. Cancer incidence and mortality patterns in Europe: estimates for 40 countries and 25 major cancers in 2018. Eur J Cancer 2018;103:356-87.

2 Sociedad Española de Oncología Médica. Las cifras del cáncer en España. Madrid 2019 https://seom.org/images/SEOM_cifras_ cancer_2019.pdf

3 Zomkowski K, Cruz de Souza B, Pinheiro da Silva F, et al. Physical symptoms and working performance in female breast cancer survivors: a systematic review. Disabil Rehabil 2018;40:1485-93.

4 Derakhshan MK, Karbassian MH. Psychiatric and psychosocial aspects of breast cancer diagnoses and treatments. In: Mehdipour P, ed. Cancer genetics and psychotherapy. Cham: Springer International Publishing, 2017.

5 Lam WWT, Shing YT, Bonanno GA, et al. Distress trajectories at the first year diagnosis of breast cancer in relation to 6 years survivorship. Psychooncology 2012;21:90-9.

6 Faller H, Schuler M, Richard M, et al. Effects of psycho-oncologic interventions on emotional distress and quality of life in adult patients with cancer: systematic review and meta-analysis. $J$ Clin Oncol 2013;31:782-93

7 Giese-Davis J, Collie K, Rancourt KMS, et al. Decrease in depression symptoms is associated with longer survival in patients with metastatic breast cancer: a secondary analysis. $J$ Clin Oncol 2011;29:413-20.

8 Dilworth S, Higgins I, Parker V, et al. Patient and health professional's perceived barriers to the delivery of psychosocial care to adults with cancer: a systematic review. Psychooncology 2014;23:601-12.

9 Sanchez MA, Rabin BA, Gaglio B, et al. A systematic review of eHealth cancer prevention and control interventions: new technology, same methods and designs? Transl Behav Med 2013;3:392-401.

10 Triberti S, Savioni L, Sebri V, et al. eHealth for improving quality of life in breast cancer patients: a systematic review. Cancer Treat Rev 2019;74:1-14.

11 Lleras de Frutos M, Casellas-Grau A, Sumalla EC, et al. A systematic and comprehensive review of Internet use in cancer patients: psychological factors. Psychooncology 2020;29:1-10.

12 Urech C, Grossert A, Alder J, et al. Web-Based stress management for newly diagnosed patients with cancer (stream): a randomized, Wait-List controlled intervention study. J Clin Oncol 2018;36:780-8.

13 Carpenter KM, Stoner SA, Schmitz K, et al. An online stress management workbook for breast cancer. J Behav Med 2014;37:458-68.

14 van den Berg SW, Gielissen MFM, Custers JAE, et al. BREATH: WebBased Self-Management for Psychological Adjustment After Primary Breast Cancer--Results of a Multicenter Randomized Controlled Trial. $J$ Clin Oncol 2015;33:2763-71.

15 Loiselle CG, Edgar L, Batist G, et al. The impact of a multimedia informational intervention on psychosocial adjustment among individuals with newly diagnosed breast or prostate cancer: a feasibility study. Patient Educ Couns 2010;80:48-55. 
16 Ryhänen AM, Rankinen S, Siekkinen M, et al. The impact of an empowering Internet-based breast cancer patient pathway program on breast cancer patients' clinical outcomes: a randomised controlled trial. J Clin Nurs 2013;22:1016-25.

17 Kim SC, Shaw BR, Shah DV, et al. Interactivity, presence, and targeted patient care: mapping e-health intervention effects over time for cancer patients with depression. Health Commun 2019;34:162-71.

18 Owen JE, Klapow JC, Roth DL, et al. Randomized pilot of a selfguided Internet coping group for women with early-stage breast cancer. Ann Behav Med 2005;30:54-64.

19 Chee W, Lee Y, Im E-O, et al. A culturally tailored Internet cancer support group for Asian American breast cancer survivors: a randomized controlled pilot intervention study. $J$ Telemed Telecare 2017;23:618-26.

20 Batenburg A, Das E. Emotional approach coping and the effects of online peer-led support group participation among patients with breast cancer: a longitudinal study. J Med Internet Res 2014;16:e256.

21 Lleras de Frutos M, Medina JC, Vives J, et al. Video conference vs face-to-face group psychotherapy for distressed cancer survivors: a randomized controlled trial. Psychooncology 2020;29:1995-2003.

22 Ochoa-Arnedo C, Flix-Valle A, Medina JC, et al. Programa E-Health ICOnnecta't: un ecosistema para fomentar el bienestar en cáncer a través de la propuesta europea ONCOMMUN. Psicooncología 2020;17:41-58.

23 Eldridge SM, Chan CL, Campbell MJ, et al. Consort 2010 statement: extension to randomised pilot and feasibility trials. $B M J$ 2016;355:i5239.

24 IBM Corporation. IBM SPSS statistics for windows, version 24.0 2016.

25 Jansen F, Krebber AMH, Coupé VMH, et al. Cost-Utility of stepped care targeting psychological distress in patients with head and neck or lung cancer. J Clin Oncol 2017;35:314-24.

26 Ochoa Arnedo C, Casellas-Grau A. Positive psychotherapy in cancer: facilitating posttraumatic growth in assimilation and accommodation of traumatic experience. In: Martin CR, Preedy VR, Patel VD, eds. Comprehensive guide to post-traumatic stress disorder. New York: Springer Verlag, 2016: 2133-49.

27 Ochoa Arnedo C, Sumalla E, Maté J. Psicoterapia Positiva Grupal en Cáncer. Hacia Una Atención Psicosocial integral del Superviviente de Cáncer. Psicooncologia 2010;7:7-34.

28 Ochoa C, Casellas-Grau A, Vives J, et al. Positive psychotherapy for distressed cancer survivors: posttraumatic growth facilitation reduces posttraumatic stress. Int $\mathrm{J}$ Clin Health Psychol 2017:17:28-37.

29 Ochoa-Arnedo C, Casellas-Grau A, Lleras M, et al. Stress management or post-traumatic growth facilitation to diminish distress in cancer survivors? A randomized controlled trial. J Posit Psychol 2020;74:1-11.

30 Zigmond AS, Snaith RP. The hospital anxiety and depression scale. Acta Psychiatr Scand 1983;67:361-70.

31 Requena GC, Martín XP, Baró MS. Discriminación del malestar emocional en pacientes oncológicos utilizando La escala de ansiedad Y depresión hospitalaria (HADS). Ansiedad y Estres 2009;15:217-29.

32 Blevins CA, Weathers FW, Davis MT, et al. The posttraumatic stress disorder checklist for DSM-5 (PCL-5): development and initial psychometric evaluation. J Trauma Stress 2015;28:489-98.
33 American Psychiatric Association. Dsm-5 classification. in: diagnostic and statistical manual of mental disorders, 5th edition. Arlington, VA: American Psychiatric Publishing, Inc, 2013.

34 Tedeschi RG, Calhoun LG. The posttraumatic growth inventory: measuring the positive legacy of trauma. $J$ Trauma Stress 1996;9:455-71.

35 Costa-Requena G, Gil F. Posttraumatic stress disorder symptoms in cancer: psychometric analysis of the Spanish posttraumatic stress disorder Checklist-Civilian version. Psychooncology 2010;19:500-7.

36 Pat-Horenczyk R, Perry S, Hamama-Raz Y, et al. Posttraumatic growth in breast cancer survivors: constructive and illusory aspects. J Trauma Stress 2015;28:214-22.

37 Ochoa Arnedo C, Sánchez N, Sumalla EC, et al. Stress and growth in cancer: mechanisms and psychotherapeutic interventions to facilitate a constructive balance. Front Psychol 2019;10:1-12.

38 Sumalla EC, Ochoa C, Blanco I. Posttraumatic growth in cancer: reality or illusion? Clin Psychol Rev 2009;29:24-33.

39 Horvath AO. An exploratory study of the working alliance : its measurement and relationship to therapy outcome. Diss Abstr Int Sect A Humanit Soc Sci 1981;42:2503.

40 Horvath AO, Greenberg LS. Development and validation of the working alliance inventory. J Couns Psychol 1989;36:223-33.

41 Horvath AO, Greenberg LS. The development of the working alliance Inventory. In: Greenberg LS, Pinsof WM, eds. Guilford clinical psychology and psychotherapy series. The psychotherapeutic process: a research handbook. New York: Guilford Press, 1986: 529-56.

42 Bordin ES. The generalizability of the psychoanalytic concept of the working alliance. Psychotherapy: Theory, Research \& Practice 1979;16:252-60.

43 Andrade-González N, Fernández-Liria A. Spanish adaptation of the working alliance inventory (WAI). psychometric properties of the patient and therapist forms (WAI-P and WAI-T). Analesps 2015;31:524.

44 EuroQol Research Foundation. EQ-5D-3L user guide, 2018. Available: https://euroqol.org/publications/user-guides/

45 Badia X, Schiaffino A, Alonso J, et al. Using the EuroQol 5-D in the Catalan general population: feasibility and construct validity. Qual Life Res 1998;7:311-22.

46 Kripalani S, Risser J, Gatti ME, et al. Development and evaluation of the adherence to Refills and medications scale (arms) among low-literacy patients with chronic disease. Value Health 2009;12:118-23.

47 González-Bueno J, Calvo-Cidoncha E, Sevilla-Sánchez D, et al. Traducción Y adaptación transcultural al español del cuestionario arms para La medida de la adherencia en pacientes pluripatológicos. Atención Primaria 2017;49:459-64.

48 Brooke J. SUS-A quick and dirty usability scale. In: Jordan PW, Thomas B, McLelland I, et al, eds. Usability evaluation in industry. London, UK: Taylor and Francis Ltd, 1996: 189-94.

49 Raudenbush SW, Bryk AS, Cheong YF, et al. HLM 7 for Windows [Computer software] 2011.

50 Ochoa-Arnedo C, Flix-Valle A, Casellas-Grau A, et al. An exploratory study in breast cancer of factors involved in the use and communication with health professionals of Internet information. Support Care Cancer 2020;28:4989-96. 